\title{
Microwave conductivity investigations of plastically deformed silicon
}

\author{
By M. Brohl $\dagger$, M. Dressel $\ddagger$, H. W. Helberg $\ddagger$ \\ and H. Alexander $\dagger$
}
† Abteilung für Metallphysik, II. Physikalische Institut, Universität Köln, F.R. Germany ¥III. Physikalische Institut, Universität Göttingen, and Sonderforschungsbereich, 126 Göttingen/Clausthal, F.R. Germany

[Received 30 October 1988\$ and accepted 16 February 1989]

\begin{abstract}
We present new results on microwave continuous-wave (c.w.) conductivity investigations of plastically deformed floating-zone (FZ) silicon single crystals. Ntype samples with various doping and deformation levels were investigated by the cavity perturbation technique. The anisotropy and absolute values turn out to be very sensitive to experimental conditions but on the whole the one-dimensional nature of dislocation conduction is confirmed. The c.w. anistropy follows the respective dislocation structure anisotropy closely. For clear results, care has to be taken of the number of deep point-defect centres also introduced by deformation. Furthermore the existence of a low-temperature conducting state is shown for a Czchrochalski $(\mathrm{CZ})$ sample annealed at $650^{\circ} \mathrm{C}$ containing rod-like defects.
\end{abstract}

\section{§1. INTRODUCTION}

The plastic deformation of silicon introduces electrically active centres into the crystal, most of which are localized deep acceptors. From electron paramagnetic resonance (EPR) spectroscopy, several types of point-defect clusters have been identified (Weber and Alexander 1977, Brohl, Kisielowski-Kemmerich and Alexander 1987). Possibly some of the deep centres reside in the core of (partial) dislocations, for example as singularities (such as jogs, kinks on reconstruction defects) or as impurity atoms and vacancies.

Moreover, several experimental results indicate the existence of deformationinduced shallow levels in addition to the deep levels. Photoluminescence reveals dislocation-related donor-acceptor pair recombination, the transition energies varying from $E_{\mathrm{g}}-165 \mathrm{meV}$ to $E_{\mathrm{g}}-217 \mathrm{meV}$ (where $E_{\mathrm{g}}$ is the width of the energy gap) (Sauer, Kisielowski-Kemmerich and Alexander 1986). Infrared absorption in the energy range $170 \mathrm{meV}$ below the fundamental absorption edge (Bazhenov and Krasil'nikova 1986) can be attributed to similar effects.

Those phenomena seem to be related to states split off the band edges by the elastic strain field of dislocations. On account of the translational symmetry along the dislocation line, these split-off states are assumed by several researchers to form onedimensional bands (Claesson 1979, Teichler 1979). The observed nearly temperatureindependent microwave (continuous-wave (c.w.)) conductivity has been attributed in some cases to these so-called deformation potential bands (Kveder, Osip'yan, Sagdeev,

$\S$ Received in final form 22 December 1988. 
Shalynin and Zolotukhin 1985), and in some cases to deep-lying dislocation-related states (Osip'yan 1981).

In this paper we report on some new results of c.w. conductivity measurements in silicon where we focus on defined control of deformation-induced deep levels due to point defects in the bulk in order to avoid electrically competing effects.

\section{§2. EXPERIMENTAL DETAILS AND RESULTS}

Table 1 lists the FZ silicon samples used in this study. The compression axis is the [213] direction. The crystals are deformed in one step at $800^{\circ} \mathrm{C}$ under a low shear stress in the main glide system up to a compressive strain $\Delta l / l$ of $1.6 \%$ (for one sample it was $5 \%$ ) in an argon gas atmosphere. Most of the crystals are annealed at $800^{\circ} \mathrm{C}$ for $16 \mathrm{~h}$ in order to anneal out most of the residual EPR- and deep-level-transient-spectroscopy (DLTS)-active centres. The integral concentration of the latter in such treated samples is below $5 \times 10^{13} \mathrm{~cm}^{-3}$.

Dislocations in samples deformed in this way are irregularly shaped; most of them are aligned as edge dipoles in the [211] direction perpendicular to the main Burgers vector $\mathbf{b}=[011]$ in the primary glide plane (111) (fig. $1(a)$ ).

A short high-stress, low-temperature deformation leads to regular dislocation structures. Dislocations will be constrained to the $\langle 011\rangle$ Peierls valleys in (111), so forming dissociated $60^{\circ}$ and screw dislocations (fig. $1(b)$ ). The number of screw dislocations drastically increases, surpassing in some cases that of $60^{\circ}$ dislocations. The number of deep levels introduced by this step should not exceed $5 \times 10^{13} \mathrm{~cm}^{-3}$ (except for sample $D$ in which the higher dislocation density leads to a higher point-defect production in the second deformation step.

Furthermore one undeformed crystal of $\mathrm{CZ}$ material $\left([\mathrm{P}]=5 \times 10^{14} \mathrm{~cm}^{-3}\right)$, annealed for $120 \mathrm{~h}$ at $650^{\circ} \mathrm{C}$ was investigated. By such a treatment $\langle 011\rangle$-oriented rodlike defects are introduced into the crystal. According to some workers the defects are accompanied by dislocation dipoles and therefore by elastic deformation fields (Bourret 1985). Recently c.w. conductivity of such crystals was assumed to be responsible for quality losses in EPR experiments but no quantitative measurements were performed (Babich, Baran, Bugai, Konchits and Maksimenko 1986). For most of the c.w. measurements, circular discs parallel to the (1I1) main glide plane were cut from the central part of the crystals. After mechanical and chemical polishing, the diameters of the discs were about $2-3 \mathrm{~mm}$ and their thicknesses $0.2-0.3 \mathrm{~mm}$. By the

Table 1. Samples used in this study. All samples are doped with phosphorus.

\begin{tabular}{|c|c|c|c|c|c|c|c|c|c|}
\hline \multirow[b]{2}{*}{ Sample } & \multirow{2}{*}{$\begin{array}{c}\text { Chemical } \\
\text { doping } \\
\left(\mathrm{cm}^{-3}\right)\end{array}$} & \multicolumn{3}{|c|}{ Pre-deformation } & \multirow{2}{*}{$\begin{array}{c}\text { Annealing } \\
\text { time } \\
\text { at } 800^{\circ} \mathrm{C}\end{array}$} & \multicolumn{4}{|c|}{ Main deformation } \\
\hline & & $\begin{array}{c}T_{\mathrm{D}} \\
\left({ }^{\circ} \mathrm{C}\right)\end{array}$ & $\stackrel{\tau}{\stackrel{\tau}{\mathrm{MPa}})}$ & $\begin{array}{l}\Delta l / l \\
(\%)\end{array}$ & & $\begin{array}{c}T_{\mathrm{D}} \\
\left({ }^{\circ} \mathrm{C}\right)\end{array}$ & $\stackrel{\tau}{\tau}$ & $\begin{array}{l}\Delta l / l \\
(\%)\end{array}$ & $\begin{array}{l}\text { Time } \\
\text { (min) }\end{array}$ \\
\hline A & $5 \times 10^{14}$ & 800 & $12 \cdot 0$ & 1.6 & $16 \mathrm{~h}$ & - & - & - & - \\
\hline B & $5 \times 10^{14}$ & 800 & $8 \cdot 0$ & 1.6 & $16 \mathrm{~h}$ & 420 & 250 & $<0.1$ & 30 \\
\hline $\mathrm{C}$ & $5 \times 10^{14}$ & 800 & $12 \cdot 0$ & 1.6 & $30 \mathrm{~min}$ & 420 & 250 & $<0.1$ & 30 \\
\hline D & $5 \times 10^{14}$ & 800 & $12 \cdot 0$ & 5.0 & $16 \mathrm{~h}$ & 420 & 200 & $<0.1$ & 30 \\
\hline E & $5 \times 10^{15}$ & 800 & $12 \cdot 0$ & 1.6 & $16 \mathrm{~h}$ & 420 & 250 & $<0.1$ & 30 \\
\hline $\mathbf{F}$ & $2 \times 10^{13}$ & 800 & $12 \cdot 0$ & 1.6 & $16 \mathrm{~h}$ & 420 & 200 & $<0 \cdot 1$ & 30 \\
\hline
\end{tabular}


Fig. 1

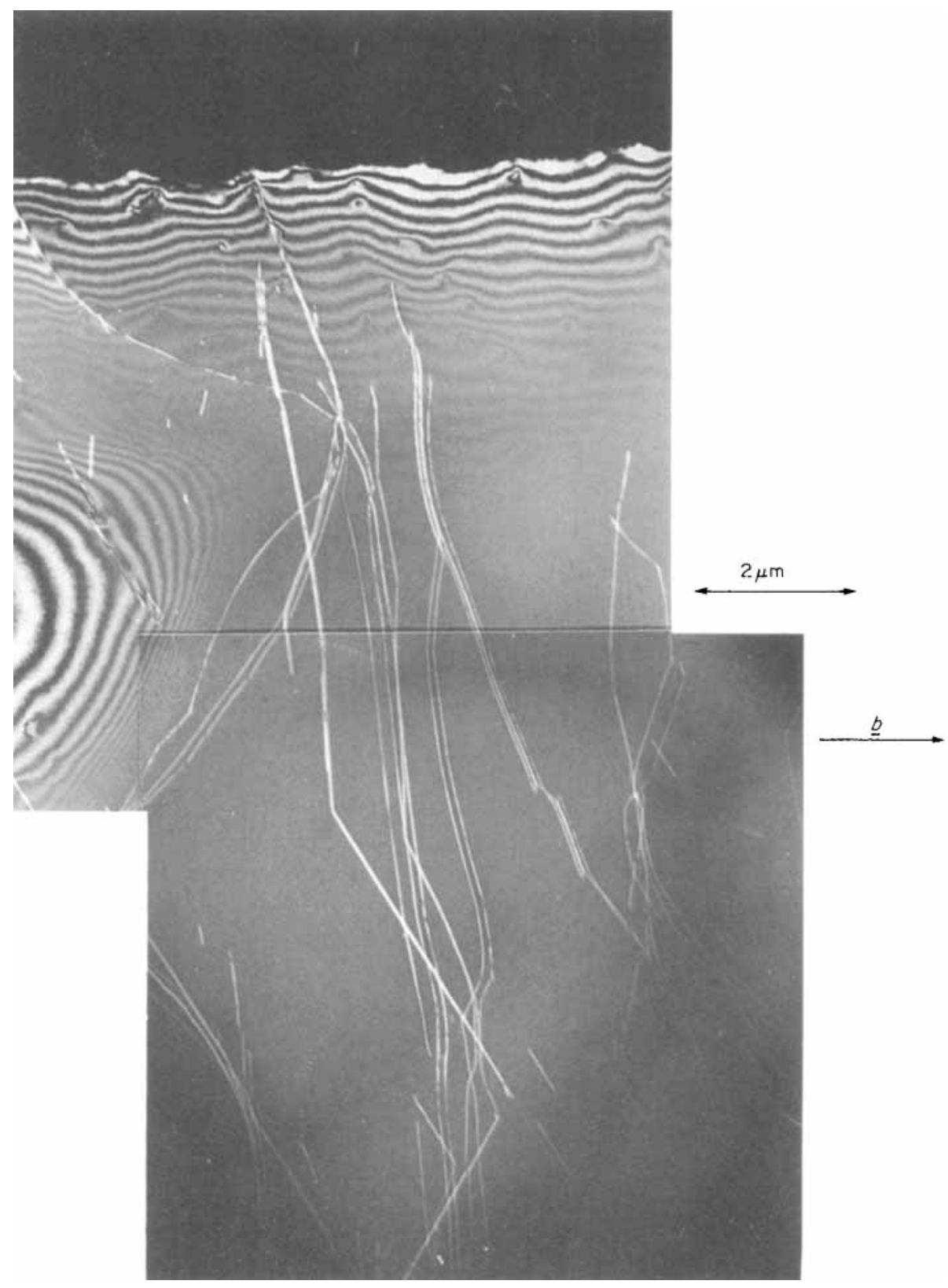

(a) 


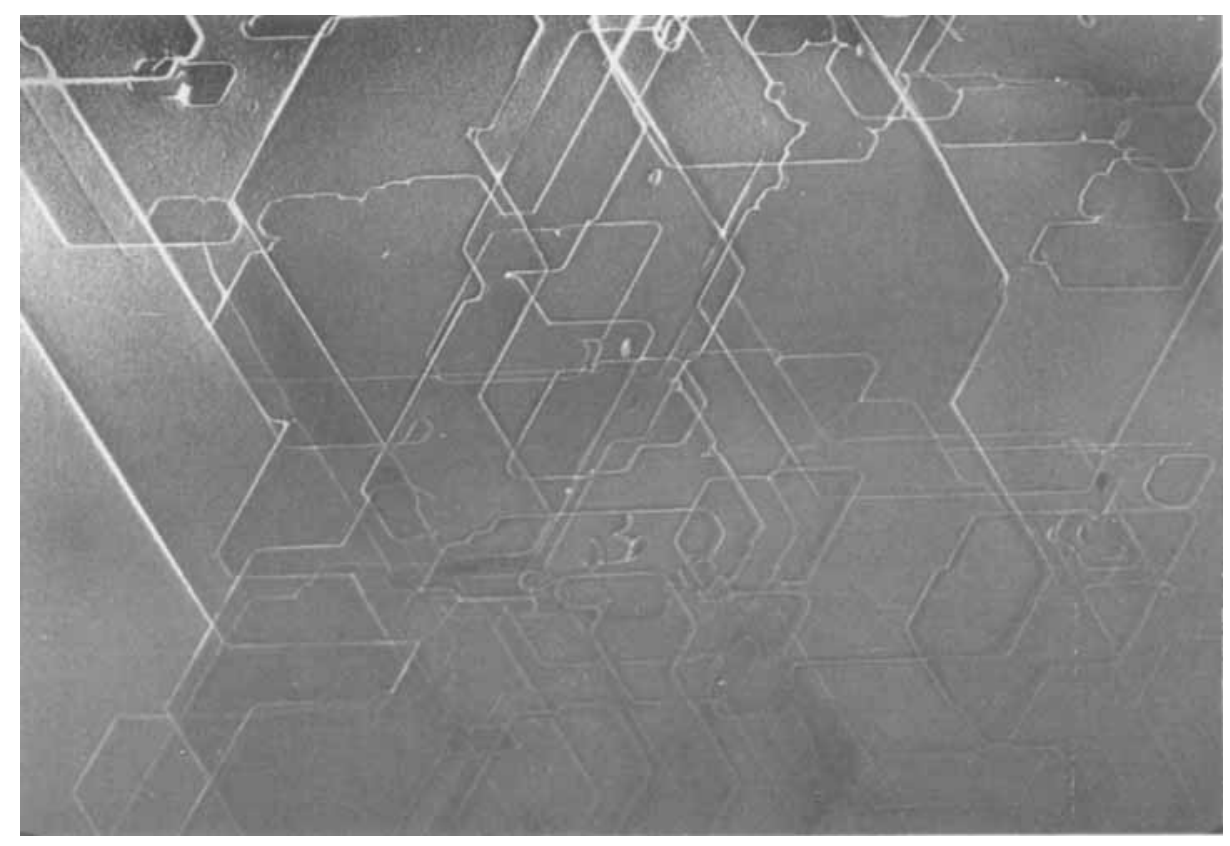

$1 \mu \mathrm{m}$

(b)

$b$

TEM dark-field contrast of dislocation structure in the main glide plane (111): (a) after predeformation at $800^{\circ} \mathrm{C}$; (b) after pre-deformation and main deformation under a high stress at $420^{\circ} \mathrm{C}$.

cavity perturbation technique as described elsewhere (Gleitz and Helberg 1985), we measured the complex permittivity at $10 \mathrm{GHz}$ as a function of temperature for different orientations of the electric field relative to the sample axes. Evaluation of the measurements followed the mathematical procedure described elsewhere (Gleitz and Helberg 1985). The absolute accuracy of the so-gained c.w. conductivity is about $50 \%$, the values for one measurement having a scatter of $5 \%$.

Figure 2(a) shows the measured c.w. conductivity at low temperatures for sample A which has only been pre-deformed. The weakly thermally activated conductivity is preferential oriented in the [21T] direction. In fig. $2(a)$ we also illustrate the influence of a second high-stress deformation step (sample B) on the low-temperature c.w. conductivity observed in comparison with specimen $A$. While as in the direction $\mathbf{E} \|[211]$ only a slight increase in $\sigma_{\text {c.w. }}$ can be detected, the conductivity parallel to $\mathbf{b}$ is drastically augmented by more than one decade. The change in c.w. conductivity therefore is closely related to that of the dislocation structure.

Rotating the electric field $\mathbf{E}$ in a plane of a sample cut perpendicular to the main glide plane, we arrive at fig. $2(b)$. Clearly, high conductivity occurs only for $E \|[211]$, which is the common direction of the two planes (111) and (011) and the direction of edge dislocations in (111). This very high anisotropy correlates with the fact, confirmed by electron micrograph investigations and by etch-pit counting, that the aboveperformed two-step deformation leads to a very pronounced single glide in the main glide system. In table 2 we summarize the anisotropies of $\sigma_{c . w .}$ and of dislocation etchpit densities (sample E).

A short annealing procedure $\left(5 \mathrm{~min}\right.$ at $420^{\circ} \mathrm{C}$ ) performed on sample $\mathrm{E}$ leads to a decrease in c.w. conductivity, the effect being more pronounced in the screw direction 
Fig. 2

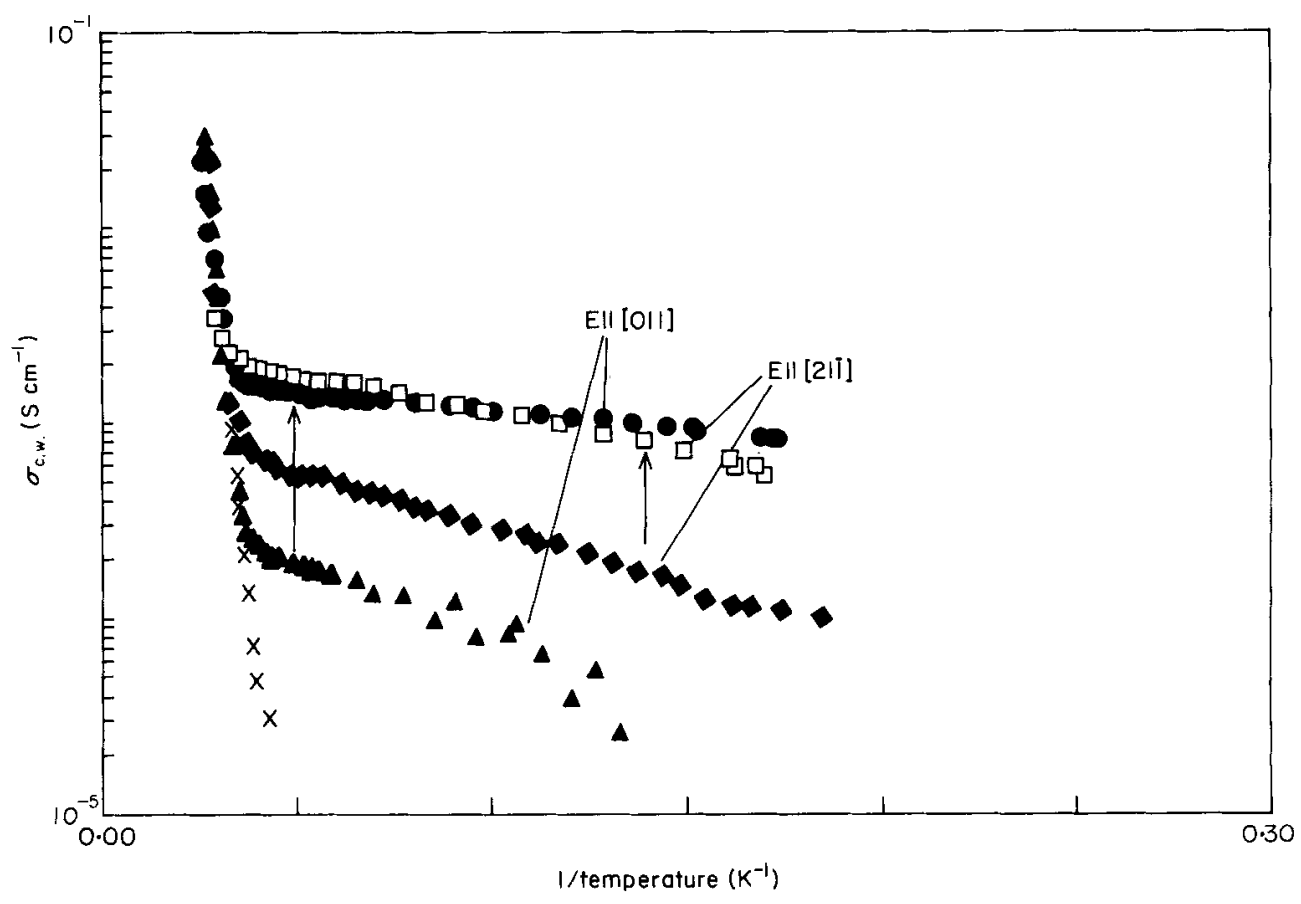

(a)

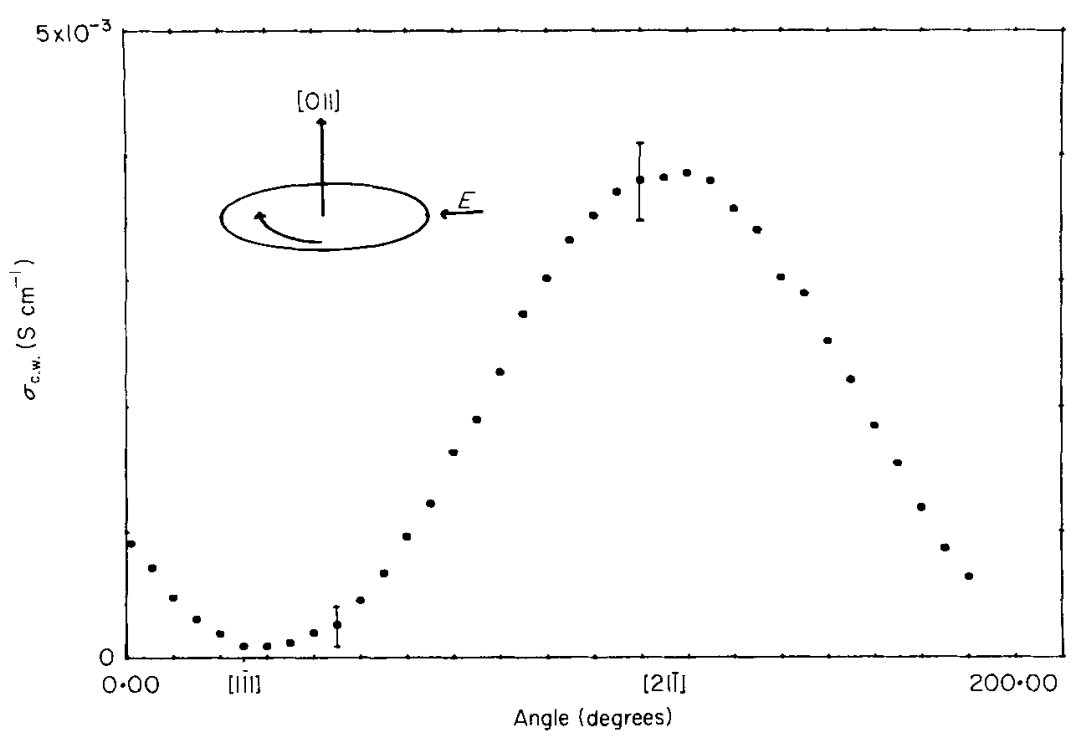

(b)

(a) C.w. conductivity against inverse temperature: $\boldsymbol{\Delta}, \bullet$, sample A (only pre-deformed); $\bullet, \square$, sample B (after high-stress low-temperature deformation); $x$, undeformed control sample. (b) C.w. conductivity against crystallographic orientation (sample E); rotation axis is the main Burgers vector $b=[011], T_{m}=12 \mathrm{~K}$. 
Table 2. Anisotropy of c.w. conductivity and dislocation structure measured for sample E. Dislocation densities are determined by etch-pit counting on the specimen faces with the specified crystallographic orientations as plane normals.

\begin{tabular}{ccc}
\hline $\begin{array}{c}\text { Crystallographic } \\
\text { orientation }\end{array}$ & $\begin{array}{c}\text { Dislocation } \\
\text { density } \\
\left(10^{7} \mathrm{~cm}^{-2}\right)\end{array}$ & $\begin{array}{c}\text { C.w. conductivity at } 12 \mathrm{~K} \\
\left(10^{-4} \mathrm{~s} \mathrm{~cm}^{-1}\right)\end{array}$ \\
\hline$[011]$ & 20 & 40 \\
{$[211]$} & 25 & 40 \\
{$[111]$} & 0.8 & 1 \\
\hline
\end{tabular}

Fig. 3

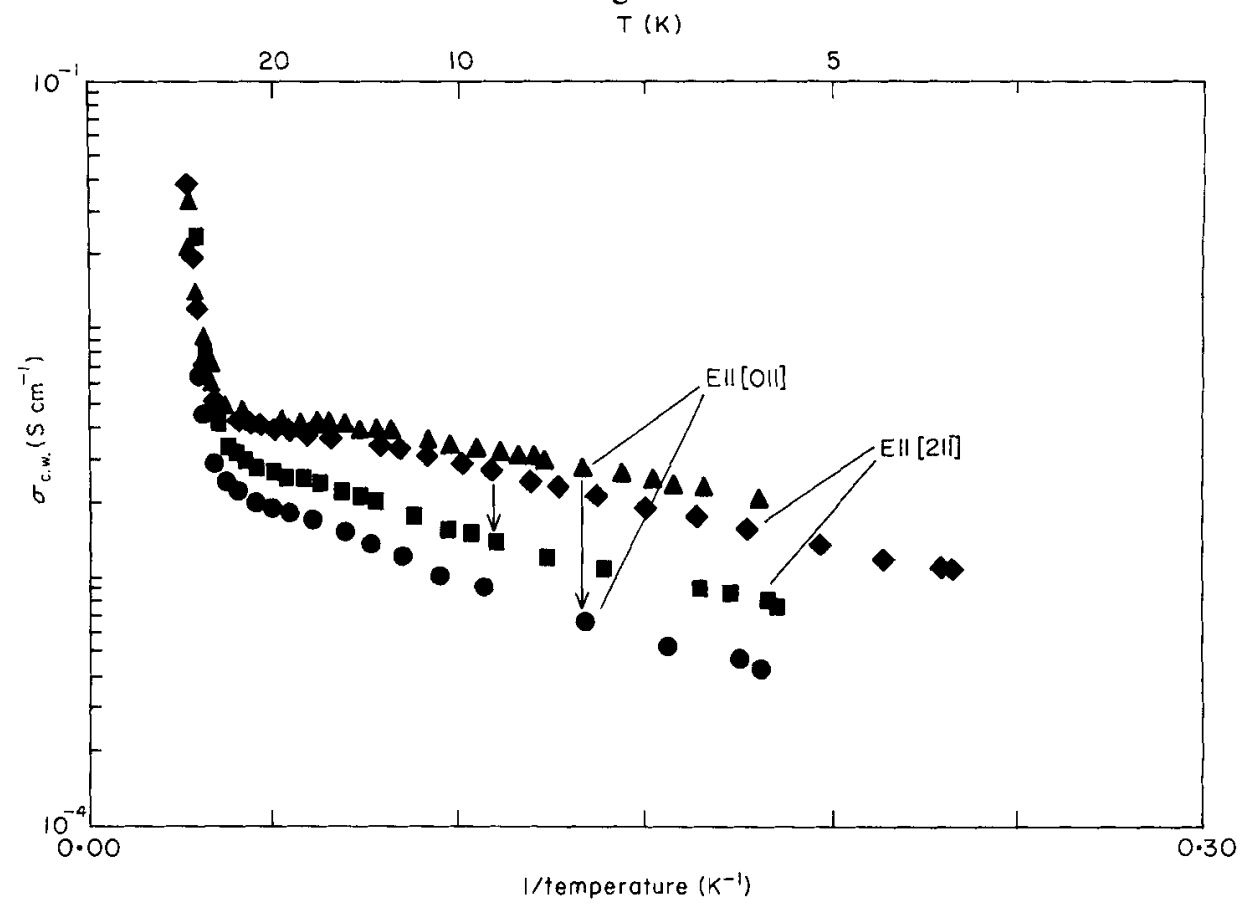

C.w. conductivity against inverse temperature: $\boldsymbol{\Lambda}, \star$, sample E; $\bullet, \mathbf{0}$, sample $\mathrm{E}$ after $5 \mathrm{~min}$ annealing at $420^{\circ} \mathrm{C}$.

$\mathbf{b}=[011]$ (fig. 3). On a microscopic scale, relaxation processes, in the form of a rounding off of dislocation loops and a narrowing of stacking-fault widths, take place. Again $\sigma_{\text {c.w. }}$. directly reflects changes in dislocation morphology. Comparing samples of different doping levels we find that the observed c.w. conductivity depends weakly on doping as long as the degree of electrical compensation of chemical donors is low but strongly drops when deformation-induced acceptor levels overcompensate the chemical doping (fig. 4). At an intermediate doping level (samples B, C and D), the results are therefore very sensitive to experimental details. A reduction in annealing time after the predeformation as well as a higher dislocation density lead to a higher concentration of residual deep acceptor levels $\left(N_{\mathrm{a}} \approx(1-2) \times 10^{14} \mathrm{~cm}^{-3}\right.$ as can be confirmed by DLTS). Therefore the crystals will be partially compensated, which results in a decrease in c.w. conductivity. 
Fig. 4

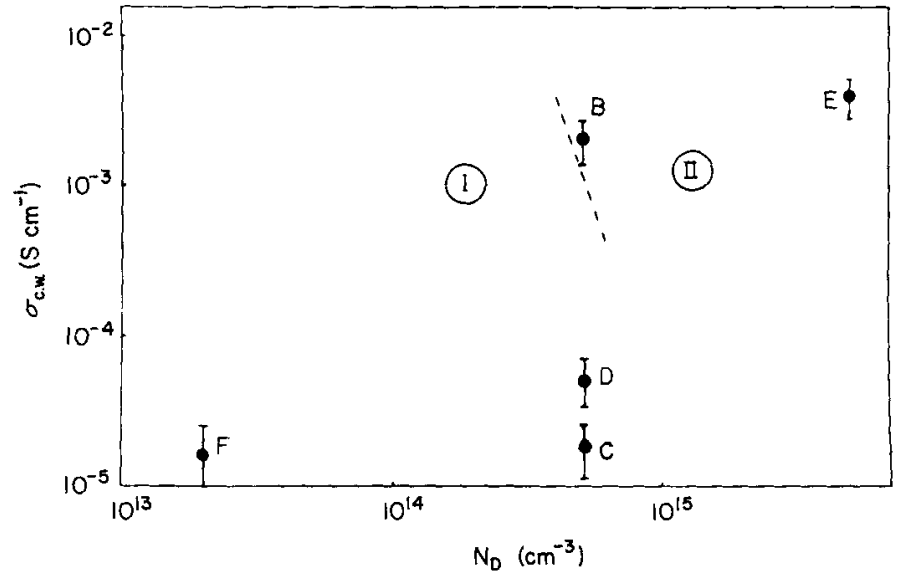

C.w. conductivity at $15 \mathrm{~K}$ for $\mathbf{E}$ parallel to $b=[011]$ against different chemical doping levels: $\mathbf{I}$, regime of strong sample compensation; II, regime of weak sample compensation.

Fig. 5

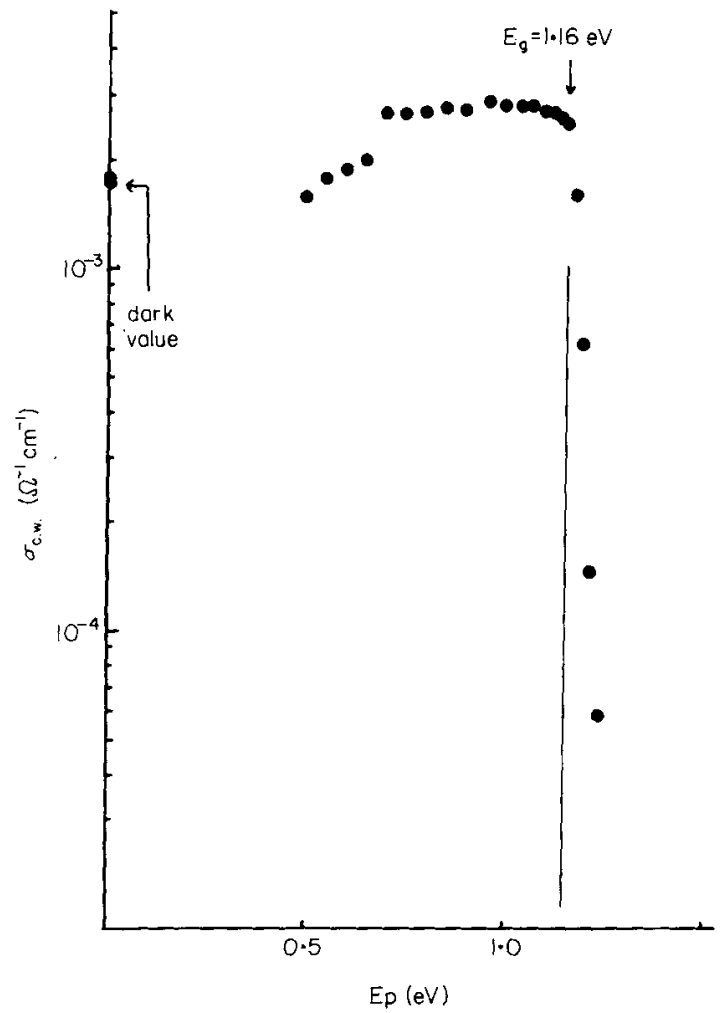

C.w. photoconductivity against incident photon energy at $9 \mathrm{~K}$ for sample $\mathrm{B}(\mathbf{E} \| \mathbf{b})$ : the vertical line indicates the gap energy. 
Fig. 6

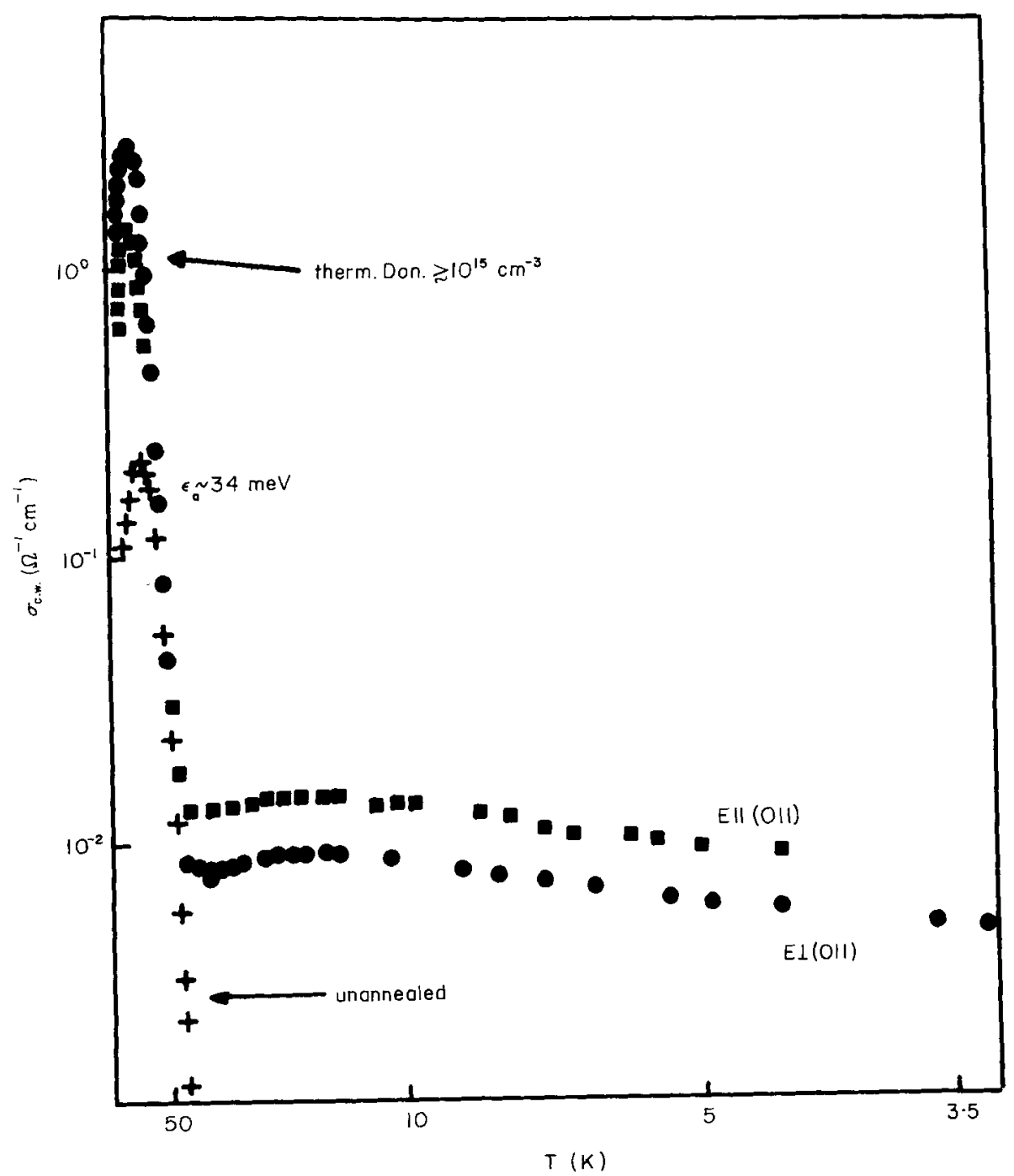

C.w. conductivity against inverse temperature for one undeformed $\mathrm{CZ}$ silicon sample annealed at $650^{\circ} \mathrm{C}$ for $120 \mathrm{~h}: \mathrm{C}, \mathrm{E}$ parallel to one of the $\langle 011\rangle$ directions;, parallel to one of the $\langle 211\rangle$ directions vertical to the above $\langle 011\rangle$ direction. $[\mathrm{O}] \gtrsim 10^{18} \mathrm{~cm}^{-3}$.

The results of photoconductivity measurements on sample B are seen in fig. 5 where $\sigma_{\text {c.w. }}(h v)$ is shown for $T_{\mathrm{m}}=9 \mathrm{~K}(\boldsymbol{E} \| \mathbf{b})$. An increase in the incident photon energy leads to a small midgap excitation $(h v \approx 0.65 \mathrm{eV}$ ) but band-to-band excitation ( $h v \geqslant 1.16 \mathrm{eV}$ ) totally destroys the dark conductivity. This last effect is not so pronounced in the other investigated samples.

Finally we state that the undeformed but annealed $\mathrm{CZ}$ crystal shows a nearly temperature-independent c.w. conductivity below $40 \mathrm{~K}$ (fig. 6). Of course, owing to the statistical distribution of the rod-like defects in all $\langle 011\rangle$ directions, there is no marked anisotropy. At higher temperatures, activation from the new thermal donors (Gaworzewski and Schmalz 1983) into the conduction band can be observed. 


\section{§3. Discussion}

According to our observations, low-temperature c.w. conductivity in plastically deformed silicon strongly depends on the detailed conditions of the experimental investigations. The anisotropy closely relates to the special well defined dislocation morphology, thus confirming the one-dimensional character of dislocation conduction in accordance with earlier publications (Osip'yan 1981, Gleitz and Helberg 1985).

Analysing our results in terms of band terminology, we have to distinguish between mobility and carrier concentration effects. The former are mainly determined by the dislocation structure, the latter by the amount of 'effective doping' of dislocations. A rough estimate can be gained of the number of carriers participating in the conduction process by means of EPR spectroscopy on the neutral phosphorus hyperfine signal $\mathbf{P}^{0}$ at low temperatures (or by carrier concentration measurements by the Hall effect at higher temperatures). In the absence of point defects acting as competing acceptors, the deformation-induced decrease in the $\mathbf{P}^{0}$ signal can tentatively be attributed to the formation of negatively charged dislocation lines surrounded by compensating Read cylinders. In our case (samples A and B) we find that nearly (2-3) $\times 10^{14} \mathrm{~cm}^{-3}$ electrons should be captured by dislocations. Regarding this number as an upper limit we estimate electron mobilities as $100 \mathrm{~cm}^{2} \mathrm{~V}^{-1} \mathrm{~s}^{-1}$ or higher. At very low temperatures (below about $10 \mathrm{~K}$ ), in all cases thermally activated conduction dominates the activation energies varying between 0.2 and $2 \mathrm{meV}$. At the present time, only speculations can be made about the nature of this metal-insulator transition-like behaviour. We can assume localization of electrons due to potential fluctuations along dislocation lines.

By photogeneration of electron-hole pairs the conducting dislocation states will be emptied by recombination processes; thus a drastic reduction in c.w. conductivity follows. This effect seems to be very sensitive to the explicit balance between doping and crystal compensation; here more detailed investigations are necessary.

The low-temperature conducting state in $\mathrm{CZ}$ silicon can be explained by the presence of two-dimensional charged boundaries between the silicon matrix and the annealing-induced rod-like defects. These states are postulated by workers performing admittance spectroscopy on such crystals (Henry, Pantrat and Saminadayar 1986). As in the case of dislocation-induced conductivity the high-frequency experiment makes visible a conduction mechanism well below a macroscopic percolation threshold.

\section{ACKNOWLEDGMENTS}

The authors thank Dr C. Kisielowski-Kemmerich for fruitful discussions and for the DLTS investigations and Dr H. Gottschalk for the TEM analysis. They are also grateful to the Deutsche Forschungsgemeinschaft for financial support.

\section{REFERENCES}

Babich, V. M., Baran, H. P., Bugai, A. A., Konchits, A. A., and Maksimenko, V. M., 1986, JETP Lett., 44, 661.

Bazhenov, A. V., and Krasil'Nikova, L. L., 1986, Soviet Phys. solid St., 28, 128.

BoURRET, A., 1985, Proceedings of the 13th International Conference on Defects in Semiconductors, Coronado, 1984, edited by L. C. Kimmerling and J. M. Parsey (Pennsylvania: Metallurgical Society of AIME), p. 129.

Brohl, M., Kisielowski-Kemmerich, C., and AleXander, H., 1987, Appl. Phys. Lett., 50, 1733.

Claesson, A., 1979, J. Phys., Paris, 40, C6-39, and references therein.

Gaworzewski, P., and Schmalz, K., 1983, Phys. Stat. sol. (a), 77, 571.

Gleirz, A., and Helberg, H. W., 1985, Phys. Stat. sol. (a), 90, K209. 
Henry, A., Pantrat, J. L., and Saminadayar, K., 1986, Mater. Sci. Forum, 10, 985.

Kveder, V. V., Osip'yan, Yu. A., Sagdeev, I. R., Shalynin, A. I., and Zolotukhin, M. N., 1985, Phys. Stat. sol. (a), 87, 657.

OsIP'Yan, YU. A., 1981, Crystal Res. Technol., 16, 239.

Sauer; R., Kisielowski-Kemmerich, C., and AleXander, H., 1986, Phys. Rev. Lett., 57, 1472. TeIChLer, H., 1979, J. Phys. Paris, 40, C6-43, and references therein.

Werer, E., and AleXander, H., 1976, Radiation Effects in Semiconductors, Dubrovnik, 1976, edited by N. B. Urli and J. W. Corbett, Institute of Physics Conference Series No. 31 (Bristol: Institute of Physics), p. 266. 